\title{
Promotion of Birth Preparedness Plan in Zambia: Impact of Male Partner Involvement on Childbirth Preparation as Strategy to Improve Maternal Health Care
}

\author{
Article by Elvis Chipili \\ PhD Public Health, Texila American University, Zambia \\ E-mail: mpunduchipili@gmail.com
}

\begin{abstract}
Background: Every pregnant woman faces a risk of life-threatening obstetric complications. It is estimated that 398 women every year die in Zambia from pregnancy related complications out of every 100,000 live births. The primary cause of this high maternal mortality is lack of access to professional delivery care. A birth-preparedness package promotes active preparation and assists in decision-making for healthcare seeking in case of such complications. Examining the role of the family, particularly the husband, during pregnancy and childbirth is important to understanding women's access to and utilization of professional maternal health services that can improve maternal health outcomes and prevent maternal mortality. However, in Zambia, the relationship between birth preparedness and decision-making on location of birth and assistance by skilled birth attendants in regard to male involvement is one subject that has not been studied. The purpose of this study was therefore to evaluate the impact of male partner involvement in childbirth preparation and contribute to the prevailing board of evidence on male involvement in addressing the high maternal mortality.

Methods: This paper was written through desk review of key policy documents, technical reports, publications and available internet-based literature. Primary analysis included studies from randomised controlled trials (RCT) whilst secondary analysis involved studies of non-randomised control trials, observational studies descriptive studies and key government reports.

Results: The study established that women have higher chances of delivering at the health facility and access skilled delivery care if they consulted and made the decision with their spouses. The proportion of women making savings for birth preparedness plan, including savings for emergencies and transport was very low without male partner involvement whereas involvement of male partners in ANC services increased the number of women attending antenatal care (ANC) services and resulted into a higher likelihood of expectant mother receiving not only skilled delivery services but also postpartum care.

Conclusion: This study provides novel evidence about male involvement during childbirth. The findings could have important implications for program planners, who should pursue all avenues and ways to involve husbands (male partners) in maternal health interventions and assess the effectiveness of education strategies targeted at husbands. Therefore, it should be viewed that examining the role of the spouses, during pregnancy and childbirth is important to understanding women's access to and utilization of professional maternal health services that can prevent maternal mortality.
\end{abstract}

\section{Introduction}

In Zambia, a significant and big burden of women's ill health in the childbearing group is due to pregnancy and child birth related complications. These women are in the productive age group of 15 to 49 years. It is estimated that 398 women every year die from pregnancy related complications out of every 100,000 live births. The lifetime risk of a woman dying from a pregnancy related complication is 1:20. This means that a pregnant woman in Zambia has a twenty times chance of developing and dying from pregnancy related complication $(\mathrm{MoH}, 2010)$. Over fifty percent of these maternal deaths are attributed to direct causes of postpartum haemorrhage, sepsis, obstructed labour, abortion, and eclampsia. Majority of these women who die from pregnancy related complications die either at home or on their way to the hospital. The largest contributor to this high number of 
maternal deaths is the delay in deciding to seek maternal and obstetrical care at both household and community levels. This delay by women in deciding to seek medical and obstetrical care even in an event of pregnancy complications is associated with lack of advance planning and preparation for childbirth on the part of the expectant family (MoH, 2010).

In trying to address the problems associated with the mentioned community delay, numerous groups implementing Safe Motherhood Programmes have since made birth preparedness and complication readiness a common strategy. The understanding of this strategy is that it motivates the family to plan to have a skilled provider at every birth. If the woman and partner make the decision to seek care before the onset of labour and they successfully follow through, with this plan, the woman will reach the care before developing any potential complications during child birth. If decisions are made in advance and various alternatives identified, regarding where to go, how to get there and how it will be paid for, delays can be avoided because the family will be prepared and risks to the mother and / or the baby can be reduced (JHPIEGO, 2004).

A birth preparedness and complication readiness plan include identification of following elements: knowledge of danger signs, the desired place of birth, the preferred birth attendant, the location of the closest appropriate care facility, funds for birth-related and emergency expenses, a birth companion, support in looking after the home and children while the woman is away, transport to a health facility for the birth, transport in the case of an obstetric complication and identification of compatible blood donors in case of emergency. Birth Preparedness reduces delays in receiving appropriate care.

Zambia among other African countries has gender power relations skewed in favour of men. Men yield a lot of power in the home and society and are regarded as the key gate keepers to decisions relating to issues of reproductive health and childbirth. It is basically men who decide when to have children and how many children a couple should have and too often these decisions may be devoid of partner consultation (Nzioka, 2001).

The role of men in the reproductive decisions of their partners has always been recognized as a factor that could either promote or hinder women's participation in Safe Motherhood programmes. Men are considered as key decision-makers in maternal and newborn care-seeking behaviours. They need to understand the needs, risks and danger signs of pregnancy, childbirth and postpartum periods to support women. Promotion of the role of men as partners and fathers is essential for their involvement and support. Yet, childbirth preparation programmes in the district are traditionally designed to interact with women and generally do not address men.

In the last decade, attention to the role of men in reproductive health has drastically increased. It is now widely recognised that men are key agents where a wide range of sexual and reproductive health practices are concerned (Bruijn, 2004). Literature has shown that healthy outcomes of pregnant women and their new borns, whether positive or negative are determined largely by decisions made by the woman's partner and the family and within the household. Increased involvement by men in fatherhood can benefit men, as well as women and children, in the form of better health. For example, men can give important psychological and emotional support to the woman during pregnancy and delivery. This, in turn, can reduce pain, panic and exhaustion during delivery (WHO, 2010). Men's involvement in maternal and child health programmes can reduce maternal and child mortality during pregnancy and labour by being prepared for example, for obstetric emergencies. The increased involvement in fatherhood can also benefit men's own health and wellbeing. For example, men who have been recognised in their new position as fathers and experienced emotional support during the pregnancy have better physical and mental health. Ntabona (2001), gives an assumption that for all the steps leading to maternal survival defined in the mother- baby package, there is always a man standing by the side of every woman knocking at the gate before during and after each pregnancy.

Reproductive health practitioners have also long recognised that the failure to target men in programmes has weakened the impact of reproductive health programmes since men significantly influence their partners' reproductive health, decision making and use of health resources (WHO, 2001). Men who are educated about reproductive health issues are more likely to support their partners in contraceptive use; they are likely to use contraception themselves and demonstrate 
greater responsibility for their children (Mehta, 2001). Furthermore, it is argued that one of the challenges for men's participation in reproductive health was the untapped potential to help reduce maternal mortality, as there was clearly a potential for much greater role of men in safe motherhood initiatives. Men could help in safe motherhood by providing resources, and transport for ANC and accompany women, by arranging for skilled attendance during delivery, by knowing the danger signs of complications and avoiding delays in decision making and transport; by ensuring good nutrition, rest and alleviating women's workload during pregnancy and postpartum as well as related physical, financial and emotional support. It is this regulating influence that would significantly improve mother's access to maternal health. In birth preparedness, it would result into availability of finances, transport in emergency situations and could bring an end to community based delays in birth preparation plan (Nzioka, 2001). Although male involvement in safe motherhood, may appear to be an easy topic (and may appear easy in practice to roll out, yet difficult to achieve), it is still a new topic compared to other areas of sexual and reproductive health such as family planning, prevention and management of STI, including HIV, and prevention and management of sexual and gender based violence (Mehta, 2001).

In Zambia, Much of the publications are based on the role of men in the prevention of HIV/AIDS transmission and sexually transmitted illnesses. There are no much published research papers on the role of men in childbirth preparation that have been done in Zambia that could be referred to and cited. One study (Groenewold et al, 200) was done in one Provincial Capital (Ndola) of Zambia and looked at gender and the roles of men in reproductive health. The findings were that men's dominance in sexual matters was a barrier to women's health protection behaviour and that women's empowerment in these matters was not very meaningful unless accompanied by related focus on change in male attitudes and behaviour (Groenewold et al, 2004).

The study observed that men play an important role in the reproductive health of women; either within marriage or consensual union, or within sexual relations of casual or commercial nature. The involvement of men covers issues like avoiding unwanted pregnancies, and the use of family planning methods, transmission of STI's, support in seeking care, gender based violence and power, communication and decision making in sexual and reproductive health matters (Groenewold et al, 2004). Men are thus seen as important representatives of their partner's social environment, and through their behaviour and expectations the gender system exerts influence on the reproductive health of women. They are socially and economically dominant especially in most African societies; they exert a strong influence over their wives, determining the timing and conditions of sexual relations, family size, and access to health care. This situation make men critical partners for the improvement of maternal health and reduction of maternal mortality (Iliyasu et al, 2010)

However, despite all these explanations on how companionship during labour can improve reproductive health and delivery outcomes, including a reduced need for caesarean section, much of these efforts nevertheless, do not yet offer clear cut guidelines on how far the partner or husband's participation can go. In spite of the recognition, overt programmatic action has not been taken to find ways to encourage their involvement as partners in Safe Motherhood programmes beyond the provision of condoms and information (Wynter and Hamilton, 2001).It has also been observed that male involvement discussions had developed mainly around contraceptive use, STDs and HIV. Comparatively little research and few programmes focus on men's relationship to maternal health (Horstman, 2004).

This desk review would therefore add on to the literature and knowledge on the impact of men in childbirth preparation as the country strives towards achieving the vision of ensuring that no woman dies whilst giving birth (MoH, 2010). This knowledge can become a viable planning asset for the organisations and programmes that are currently striving to promote safe motherhood practices within the country and seek to accelerate the reduction of mothers dying from childbirth complication 


\section{Objectives}

The primary aim of this review was to evaluate the impact of male partner involvement in childbirth preparation as a strategy for the improvement of maternal health care and reduction of maternal mortality. The objectives were fourfold: 1) To determine the impact of male partner involvement in childbirth preparation on improving maternal health care, 2) determine areas of male partner involvement in childbirth preparation that has impact on improving maternal health care, 3) provide evidence based literature of the impact of male partner involvement in childbirth preparation as a strategy to improving maternal health care, 4) to identify the knowledge gap in the locally available literature of the significance of male partner involvement in childbirth preparation in reducing maternal mortality.

\section{Methods}

This paper was written through desk review of key policy documents, technical reports, publications and available internet-based literature.

\section{Types of studies}

Primary analysis included a few available randomised controlled trials (RCT) on the subject under study. Secondary analysis included non-randomised control trials, observational studies, descriptive studies and key government reports when very few RCTs were found. In order to be included in the study, the subject of male partner involvement in maternal health had to be indicated both in the title and abstract of the study paper. Reports of interventions had to be published in a book, key government publications or peer-reviewed journals and be targeted at adult men as a distinct group. Studies that reported interventions were included only if they contained evaluation data. However, in several cases, because of the lack of studies, reports were included that contained only descriptive information because they illustrated an area that was a particular focus for work with men in either reducing adverse outcomes of pregnancy or that aimed at improving pregnancy outcomes.

\section{Type of participants}

Comprised of married men. Only studies that focussed on male partners' involvement or participation in safe motherhood with preference to childbirth preparation and complication readiness were included for analysis. Studies that focussed on male partner involvement in other reproductive health related areas like HIV and STI prevention were excluded. These were excluded because they were considered to have no direct effect on maternal mortality reduction. The studies considered for analysis were purposely selected as they were considered to have the views related and necessary to answer the research question.

\section{Types of intervention/issue}

These included health educational intervention (partner education on elements of birth plan strategy) with husbands (partners), participation in birth preparation plan including provision of transport or funds for emergencies, selecting a birth location, identifying the location of the closest appropriate care facility, identifying a skilled attendant, identifying a companion for birth and for emergency and having adequate supplies for birth. The husbands were educated about their role in nutrition and health of their wives during pregnancy and their responsibility in the subsequent child rearing. They were explained in detail the physiology of pregnancy, complications of pregnancy and the possible ways and means of preventing the complications. They were also told to encourage their wives to attend the antenatal clinic of the centre as frequently as possible.

\section{The inclusion and exclusion criteria}

The table below summarises the criteria used for including and excluding the evidences considered for this study 
Table 1. The inclusion and exclusion criteria

\begin{tabular}{|c|c|c|}
\hline & Inclusion criteria & Exclusion criteria \\
\hline Population & Adult Male partner (spouse) & Young males (unmarried) \\
\hline Intervention & $\begin{array}{l}\text { Active male partner } \\
\text { Involvement in childbirth } \\
\text { plan }\end{array}$ & Lone women involvement \\
\hline $\begin{array}{l}\text { Comparative } \\
\text { Intervention }\end{array}$ & $\begin{array}{l}\text { Usual female partner lone } \\
\text { involvement (non male } \\
\text { involvement) }\end{array}$ & No comparative intervention \\
\hline Outcomes & $\begin{array}{l}\text { Joint birth plan making } \\
\text { Skilled ANC utilisation } \\
\text { outcomes } \\
\text { Skilled delivery utilisation } \\
\text { outcomes } \\
\text { Maternal and neonatal } \\
\text { mortality outcomes } \\
\text { Knowledge of maternal health }\end{array}$ & $\begin{array}{l}\text { Un specific (not numerically / } \\
\text { statistically determined) } \\
\text { maternal utilisation outcomes } \\
\text { Lack of comparison - not } \\
\text { showing relationship between } \\
\text { outcomes for intervention } \\
\text { groups and outcomes for } \\
\text { controls }\end{array}$ \\
\hline $\begin{array}{l}\text { Types of } \\
\text { studies }\end{array}$ & $\begin{array}{l}\text { Quantitative- RCT } \\
\text { Qualitative studies } \\
\text { Systematic reviews } \\
\text { Key government reports }\end{array}$ & $\begin{array}{l}\text { No inclusion of evaluation } \\
\text { data. } \\
\text { Descriptive information not } \\
\text { illustrating an area that was a } \\
\text { particular focus for work with } \\
\text { men in either reducing } \\
\text { adverse outcomes of } \\
\text { pregnancy or that aimed at } \\
\text { improving pregnancy } \\
\text { outcomes. } \\
\text { Reports of interventions not } \\
\text { published in a book, key } \\
\text { government publications or } \\
\text { peer-reviewed journals. }\end{array}$ \\
\hline
\end{tabular}

\section{Types of outcome measures}

Birth preparedness was assessed based on the number of arrangements a couple had made, including (i) saving money for delivery, (ii) arranging for a blood donor, (iii) arranging for transportation to delivery and (iv) making an emergency plan. Health care utilization indicators included whether a woman received the recommended ANC visits, delivered in a health institution, had a skilled birth attendant or attended a postpartum visit at health facility within 2 weeks of delivery. Birth outcome indicators included maternal mortality and morbidity.

\section{The search strategy}

A literature search of online databases including Medline, Cochrane Library, Cihnal, Trip index and National Research Register was conducted. When this search failed to yield more than a handful of published accounts, the search strategy was widened to include Google Search engine. The search was based on a series of search terms and topics suggested by the substantial literature around men's involvement in maternal health and reproductive health. These terms and topics included men's role in childbirth preparedness and maternal death prevention, men's role in the avoidance of unwanted pregnancies, men's role in the promotion of safe motherhood and men's involvement in fatherhood.

\section{Focused question}

The focused question that directed the search strategy of this study is stated below:

"Does male partner involvement in childbirth preparation have an impact on improving maternal health care?". 


\section{Methodological quality assessment}

Methodological quality assessment was conducted to assess the risk of bias in included studies. Study quality assessment was guided by analysis of study designs, the level of details required in the assessment, the ability to distinguish between internal validity (risk of bias) and external validity or generalisability (Centre for Reviews and Dissemination, 2008). For the RCT and Qualitative papers, quality assessment was done with the help of Critical Appraisal Skills Programme (CASP) tools (Public Health Resource Unit, 2006) for the step by step critical appraisal of the published evidences. Evidences from government and other official publications were evaluated for their credibility and validity considering their sources of publications.

\section{Results of the search strategy}

Results of the search strategy are presented in details in appendix 1.

\section{Data extraction}

Data extraction was done using the data extraction form. Full details of the data extracted are shown in appendix 4.

\section{Results}

The study results are presented in accordance with the aim and the objectives of this study: impact of male partner involvement in childbirth preparedness, maternal health care utilisation and birth outcome indicators included maternal mortality and morbidity. A high proportion of papers reviewed however, produced descriptive statistics as opposed to inferential statistics. Much of the results of this study therefore were mainly based on the descriptive statistics.

\section{Impact of male partner involvement in childbirth preparedness}

Analysis of study results took into account the fulfilment of the elements of the birth plan and included emergency savings and joint decision making.

\section{Joint decision making}

Kakaire et al (2011) in a study; Male involvement in birth preparedness and complication readiness for emergency obstetric referrals in rural Uganda found that the final decision regarding location of birth when made by the woman herself was $36 \%$ and the woman with spouse $(56 \%)$ and the woman with relative/friend (8\%). The relationships between birth preparedness and women decision-making on location of birth in consultation with spouse/friends/relatives and choosing assistance by SBAs showed statistical significance which persisted after adjusting for possible confounders (OR 1.5, 95\% CI: 1.0-2.4) and (OR 4.4, 95\% CI: 3.0-6.7) respectively.

\section{Complication and emergency readiness}

Results from a study conducted by Iliyasu et al (2010) indicated that only a low proportion of women prepared for births without the involvement of their spouses. The results indicated that few women had made some savings for emergencies (19.5\%) and transportation during labour (24.2\%). Similarly, a Ugandan study done by Kakaire et al (2011) cited above also indicated a low percentage of women (44.3\%) who made savings for any eventualities such as pregnancy complications.

\section{Impact of male involvement in maternal health care utilisation}

\section{Skilled ANC attendances}

Tweheyo (2010) in a study that looked at male partner attendance of skilled antenatal care in peri-urban Gulu district of Northern Uganda found that the level of couple attendance of skilled ANC was relatively high at $65.4 \%$ when males accompanied their wives for ANC services. This was contrasted from the results ( $42 \%$ ) obtained in Omoro county in Gulu district of the same country were the level of couple attendance of skilled ANC was relatively low. The Guru District ANC attendance levels were observed to be even much higher than the national ANC level attendances (4\%) reported by the nation's Health Management information System (HMIS) of 2008. 


\section{Skilled delivery care utilisation}

Nanjala and Wamalwa (2012) found that male partner's level of education $(\mathrm{P}=0.0000)$ and occupation ( $\mathrm{P}=0.0004)$ had an effect on his involvement in supporting the spouse to access delivery services from skilled attendants. They also found that the spouses' age and access to delivery services from skilled attendants was statistically significant $(\mathrm{P}=0.000)$. Socio-cultural factors were also found to affect the involvement of male partners in supporting their spouses to access skilled delivery services. The study established no significant statistical difference between the age of a male partner and the type of delivery (skilled or unskilled) of the spouse $(\mathrm{P}=0.4259)$. In another study, Mullany et al (2006) found that Women who received education with their husbands were nearly twice as likely as control group women to report making adequate birth preparations. This study further established that educating women together with their partners resulted into a higher likelihood of women receiving skilled postpartum care compared to women who received education alone or no education at all. Additionally a qualitative study done in rural Bangladesh that looked at husbands' involvement in delivery care utilization in rural Bangladesh established that husbands whose wives utilized professional delivery care provided emotional, instrumental and informational support to their wives during delivery and believed that medical intervention was necessary. By contrast, husbands whose wives utilized an untrained delivery care at home were uninvolved during delivery and believed childbirth should take place at home according to local traditions (Story, 2011).

\section{Impact of male involvement on maternal and neonatal outcomes}

Evidences from literature reviewed studies indicated that male involvement in childbirth preparation came along with health promoting outcomes for the expectant mothers. A European study Fatherhood and Health Outcomes in Europe by WHO (2007) established that male involvement resulted into emotional and psychological support, reduced maternal and child mortality and resulted into an improved husband and Wife communication.

\section{Discussions}

Review of literature indicates that positive or negative health outcomes of pregnant women are largely determined by a woman's partners and the family and within the household (WHO, 2010). Women and newborns need timely access to skilled care during pregnancy and child birth. Too often their access to care is impeded by delays: delays in deciding to seek care, delays in reaching care and delays in receiving care. These delays have many causes that include logistic and financial concerns as well as inadequate community and family awareness and knowledge about maternal and newborn issues (MoH, 2010). It is thus cardinal and important to understand men's behaviours and their point of view because, given the gender asymmetry present in most societies, they still have a dominant role in reproductive health related decisions and outcomes. Studying men should not be seen as contradictory to studying women; but this shows us the other side of the coin and many mirror images that enrich the gender analysis (Pandelides, 2001).

\section{Impact of male involvement on birth/emergency preparedness plan}

Improving family awareness and knowledge about maternal and newborn issues is the main purpose of a birth plan/emergency preparedness plan. The birth plan/emergency preparedness plan aims among others things at achieving an increase in care seeking behaviour and is closely linked to actions to develop transport, finances and enhance support of men (WHO, 2003). Men could help in a birth/emergency preparedness plan by providing resources, transport for ANC and accompany women. They can help in arranging for skilled attendance during delivery, by avoiding delays in decision making and transport, ensuring good nutrition, rest and alleviating women's workload during pregnancy, providing physical and emotional support (WHO, 2010).

The studies reviewed in this study indicated that there is a relationship that exists between birth preparedness and the woman's decision making of location of delivery and choice of birth attendants. An expectant woman will in one way or the other find the necessity to consult members of the family or closer associates as regards the location of the delivery place and those that will 
have to assist her deliver her child. Depending on the consultations made, she will either deliver traditionally in the house of the untrained birth attendant or at the health facility in the hands of the skilled birth attendant. The study by Kakaile and friends established that chances of her delivering at the health facility and access skilled delivery care are higher if she consulted and made the decision with the spouse (56\%), 36\% if she made the decision alone and only $8 \%$ if she consulted the relatives or friends (Kakaire et al, 2011). This finding has been acknowledged by literature that suggests that if the woman and partner make the decision to seek care before the onset of labour and they successfully follow through, with this plan, the woman will reach the care before developing any potential complications during child birth. If decisions are made in advance and various alternatives identified, regarding where to go, how to get there and how it will be paid for, delays can be avoided because the family will be prepared and risks to the mother and / or the baby can be reduced (JHPIEGO, 2004). This finding can also be a basis for the development of safe motherhood programmes that seeks to empower women and male partners with maternal health care information. It has long been established that men who are educated about reproductive health issues are more likely to support their partners in contraceptive use; they are likely to use contraception themselves and demonstrate greater responsibility for their children (Mehta, 2001).

Literature has shown that men could help in safe motherhood by providing resources, and transport for ANC and accompany women, by arranging for skilled attendance during delivery, by knowing the danger signs of complications and avoiding delays in decision making and transport; by ensuring good nutrition, rest and alleviating women's workload during pregnancy and postpartum as well as related physical, financial and emotional support (Horstman (2004). This assertion has been proven to be true in this study. A 2010 Nigerian research done in the Northern community of Nigeria by Iliyasu and friends established that the proportion of women making savings for emergencies and transport was very low in proportion to low male involvement. The researchers found that majority of pregnancies were unplanned (96\%). Only $32.1 \%$ of men ever accompanied their spouses for maternity care. There was very little preparation for skilled assistance during delivery (6.2\%), savings for emergencies (19.5\%) or transportation during labour $(24.2 \%)$. The obstetric referrals are low and contribute significantly to maternal and neonatal morbidity and mortality. With this low male participation pregnancy and childbirth has been regarded as woman's business (Kakaire, 2011). Arrangement for transport is important in the birth preparation plan. It has been established by research that men with their firm control of household resources may play a critical role in ensuring that a suitable fast transport is arranged in advance to quicken the movement of mothers to the health facility both in normal and emergency cases . The birth preparedness plan requires that the husband and wife (partner) jointly decide and arrange for transport long before time of delivery (Nzioka, 2001). In this situation, women would find it difficult to reach the obstetric referral sites and has the case is in Zambia many pregnant women faced birth complications delay in reaching decisions to seek skilled delivery care and as a consequence either died whilst labouring in their homes or died on their way to hospital whilst being transported on bicycles or ox drawn charts $(\mathrm{MoH}, 2010)$.

\section{Impact of male involvement on skilled ANC attendances}

This study has further shown that higher utilisation coverage for skilled ANC and skilled delivery services were obtained with increased male participation and vice versa. In the peri-urban Gulu district of Northern Nigeria a research conducted by Tweheyo (2010) found that involvement of male partners in ANC services increased the number of women attending ANC services to $65.4 \%$. This was higher than the ANC attendances prevailing in the other areas of the Gulu district (42\%) and far much higher than the national ANC coverage attendances (4\%). Kakaire et al (2011) conducted a similar study in the rural area of the same country and also found low skilled ANC coverage $(42.9 \%)$ where only a small proportion of husbands accompanied their wives for antenatal clinics (refer to appendix 6). The findings above agree with the findings in Malawi by Kalulanga et al (2011), that suggested that maternal health education interventions targeting both men and women have proved to increase knowledge in both men and women; increase health seeking behaviour among pregnant women; raise awareness and use of family planning (FP) in the 
postpartum period, and also increased awareness of dual protection for STIs. The study also noted that men's participation in antenatal education programmes had positive effects including increase in men's reproductive health and child health knowledge, high utilization of antenatal care services by women.

\section{Impact of male involvement on skilled delivery services}

Mullany et al (2006) in a randomized controlled trial study done in Nepal, found that Women who received education with their husbands were also nearly twice as likely as control group women to report making more birth preparations. The study further established that educating women together with their partners resulted into likelihood of women receiving not only skilled delivery services but also postpartum care compared to women who received education alone or no education at all. Additionally a qualitative study done in rural Bangladesh that looked at Husbands' involvement in delivery care utilization in rural Bangladesh established that Husbands whose wives utilized professional delivery care provided emotional, instrumental and informational support to their wives during delivery and believed that medical intervention was necessary. By contrast, husbands whose wives utilized an untrained delivery care at home were uninvolved during delivery and believed childbirth should take place at home according to local traditions (Story, 2011).

A study by Kabakyenga, 2012 in Uganda highlighted the importance of having skilled birth attendants. They noted that assistance by skilled birth attendants (SBAs) is one of the strategies aimed at reducing maternal morbidity and mortality in low-income countries. Most of the causes of maternal morbidity and mortality are preventable and attributed to the three delays; delay to make a decision to seek care, delay to reach the place of care, and delay to receive appropriate care. Having a skilled birth attendant (SBA) at every delivery has been found to markedly reduce maternal morbidity and mortality in many countries that include Malaysia and Sri Lanka. Prompt decision-making is a prerequisite for reducing delay to seek care. Shija et al, 2011 in a study that looked at challenges and opportunities in reducing maternal mortality in Tanzania, further noted that more than $80 \%$ of maternal deaths can be prevented if pregnant women access essential maternity care and assured of skilled attendance at childbirth as well as emergency obstetric care. Most of maternal deaths are caused by factors attributed to pregnancy, childbirth and poor quality of health services. About $60 \%$ of maternal deaths occur during labour, delivery and immediate postpartum period. Fifty percent of these deaths occur within the first 24 hours of delivery. Comparatively, just like in Zambia, a woman in East Africa has 1 in 12 risk of dying due to pregnancy as compared to 1 in 4,000 in northern Europe. Most complications cannot be predicted; therefore timely diagnosis with skilled personnel is important to avoid introducing harm (Shija et al, 2011).

Nanjala and Wamalwa (2012), in their study: Determinants of Male Partner Involvement in Promoting Deliveries by Skilled Attendants in Busia, Kenya found increased access to delivery services from skilled attendants where husbands accompanied their spouses for delivery care services. This was statistically even more significant with male partners higher level of education $(\mathrm{P}=0.0000)$ and for occupation $(\mathrm{P}=0.0004)$. There was also a higher relationship between spouses' age and social cultural factors and access skilled delivery services. The study established that spouses' age and access to delivery services from skilled attendants was statistically significant $(\mathrm{P}=0.000)$ and that Socio-cultural factors affect the involvement of male partners in supporting their spouses to access skilled delivery services. According to Zambian demographic health surveys (CSO, 2007), it was found that only $48 \%$ of the total women population was literate and that of men $72.7 \%$. The low literacy levels of women and also of men have an impact on the level and quality of their decision making process and may determine how such individuals and families accept and take up health care services including Safe motherhood programmes.

\section{Impact of male involvement on maternal and neonatal outcomes}

Literature has shown that Health outcome of pregnant women and their new borns, whether positive or negative are determined largely by decisions made by the woman's partner and the family and within the household (WHO, 2010). A 2007 WHO study: Fatherhood and Health 
Outcomes in Europe revealed that increased involvement by men in fatherhood can benefit men, as well as women and children, in the form of better health. For example, men can give important psychological and emotional support to the woman during pregnancy and delivery. This, in turn, can reduce pain, panic and exhaustion during delivery.

The study showed that men's involvement in maternal and child health programmes can reduce maternal and child mortality during pregnancy and labour by being prepared for example, for obstetric emergencies. The increased involvement in fatherhood can also benefit men's own health and wellbeing. For example, men who have been recognised in their new position as fathers and experienced emotional support during the pregnancy have better physical and mental health (WHO, 2007). The influence of men in decision-making has been seen in studies of family planning, sexually transmitted infections and HIV, abortion, and infertility. However, there is a relative scarcity of information on men's intentions and practices as they relate to pregnancy and childbirth, especially in Africa and Zambia in particular. Some have suggested that male partners act as obstacles when it comes to safe delivery care. However, male involvement during pregnancy and childbirth can lead to positive birth outcomes for the mother and child as well as a healthier marital relationship. A husband's positive involvement can take many forms, including transporting his wife to a qualified provider, providing household money to make that visit, giving helpful informational support during pregnancy, and offering emotional support during labour and childbirth. This means that even though men may be discouraged from being involved in matters of pregnancy and childbirth, as may be the case in certain regions like South Asia, their beliefs and perceptions might influence where and how their wives give birth. The husband's perception of social norms related to professional care is important to the type of advice and support he will give his wife during pregnancy and childbirth, which has important implications for the development of future maternal health interventions (Story, 2012).

Since gender inequalities favour men, in patriarchal societies and sexual and reproductive health decisions are made by them, there is a growing realisation that unless men are reached, reproductive health programme efforts will have limited impact. Because of unequal gender power relations, women are especially vulnerable as they are unable to negotiate changes in sexual behaviours to prevent unwanted pregnancy and to practice safer sex. Therefore, the involvement of men as responsible partners is essential (Duza, 2001).

\section{Recommendations}

The recommendations below are based on this study's findings and are supported by literature review:

1) Safe motherhood programmes should design more maternal health programs that involve men and educate them about male involvement in childbirth preparation as a strategy to improving maternal health outcomes to prevent maternal and neonatal deaths.

2) ANC services should develop strategies to empower men with knowledge about ANC so as to increase ANC attendances and in turn increase skilled deliveries.

3) Safe motherhood programmes should develop guidelines on men's involvement in childbirth preparation.

4) Specific strategies should be in place to empower women and men with complemented adequate maternal health care information for them to make informed and joint decisions of issues affecting maternal and neonatal outcomes.

\section{Application of evidence}

Studies reviewed in this desk review suggest that there is greater improvement in maternal and child health care services with male involvement. Kakaire et al (2011) and friends established that chances of a pregnant woman delivering at the health facility and access skilled delivery care are higher if she consulted and made the decision with the spouse, Iliyasu's study in Nigeria established that the proportion of women making savings for emergencies and transport was very low without male involvement, whereas Tweheyo (2010) found that involvement of male partners in ANC services increased the number of women attending ANC services to well over $65.4 \%$. Similarly, 
Mullany et al (2006) in a randomized controlled trial study done in Nepal found that Women who received education with their husbands were also nearly twice as likely as control group women to report making more birth preparations. The study further established that educating women together with their partners resulted into likelihood of women receiving not only skilled delivery services but also postpartum care compared to women who received education alone or no education at all.

Review of Zambian policy documents indicates similar behavioural patterns. For instance in Zambia it is mandatory that all pregnant women attend the four skilled ANC Visits. However, evidence suggests that about $90 \%$ of pregnant women in Zambia attend the first ANC visit. Out of these $70 \%$ manage to turn up for the fourth visit and only $43 \%$ finally manage to access the much promoted skilled delivery at a health facility. Just a small proportion of about $23 \%$ reports back after delivery for post-natal care $(\mathrm{MoH}, 2010)$. The figure below (figure1) summaries the evidence above. Locally in one of the rural districts in Zambia (Lundazi district) evidence suggest that $77 \%$ of pregnant women are accompanied by their male partners for the first ANC visit (LDHMT, 2012). No data is available for the percentage of pregnant women accompanied by males for the three subsequent visits. It is actually policy that for the first ANC visit, all pregnant women should be accompanied by their spouses to have their HIV testing as a PMTCT measure. There is need for studies to be conducted to ascertain as to whether, the high turn up of men for the first ANC visit is as a result of men's change of behaviour and commitment to ANC services or as to whether men are simply being compelled by the policy existing to avoid their partners being denied of the service. But whether, the high turn up for men in the first ANC visits is due to policy or behavioural change presents an opportunity that ANC service providers can utilise to provide the necessary information to both women and men as regards childbirth and complication readiness plan.

In the year 2010, the Zambian government launched a vigorous sensitisation campaign called the Campaign for Accelerated Reduction of Maternal Mortality in Zambia (CARMMZ). The aim of the campaign was to mobilise support for maternal health services under the theme: "Zambia Cares, no woman should die whilst giving birth" (MoH, 2010). However, despite the high numbers of organisations promoting Safe motherhood strategies at community level, and the vigorous sensitisation campaign by the government, there is hardly any specific programme that deliberately targets and addresses male involvement in childbirth preparation in the district as a measure to prevent and reduce maternal mortality. Women have continued to deliver in large numbers at home in the hands of totally unskilled birth attendants. A high number of them too continue to perish helplessly in the process of giving life within the vicinity and in the homes of their partners. Equally there is hardly a credible study done to explore male partner involvement in childbirth preparation in Zambia. There is therefore, a need to explore closely the roles of men in the whole process of child birth preparation to gain insight of the joint birth plan decision making process at family level.

There is a need for more research to look not just at the process and immediate outcomes of men's involvement, but also to have a longer term impact on the lives of both women and men. The reality is that despite the considerable rhetoric surrounding men's involvement, men are still seldom targeted and there are very few evaluations of interventions in sexual and reproductive health that address issues from a focal point of masculinity, or even an understanding of men's needs (Sternberg and Hubley, 2004). The inescapable conclusion is that in issues of male involvement in childbirth preparation and maternal deaths prevention, although perhaps no longer seen just as part of the problem, men have yet to be regarded seriously as part of the solution (Sternberg and Hubley, 2004) 


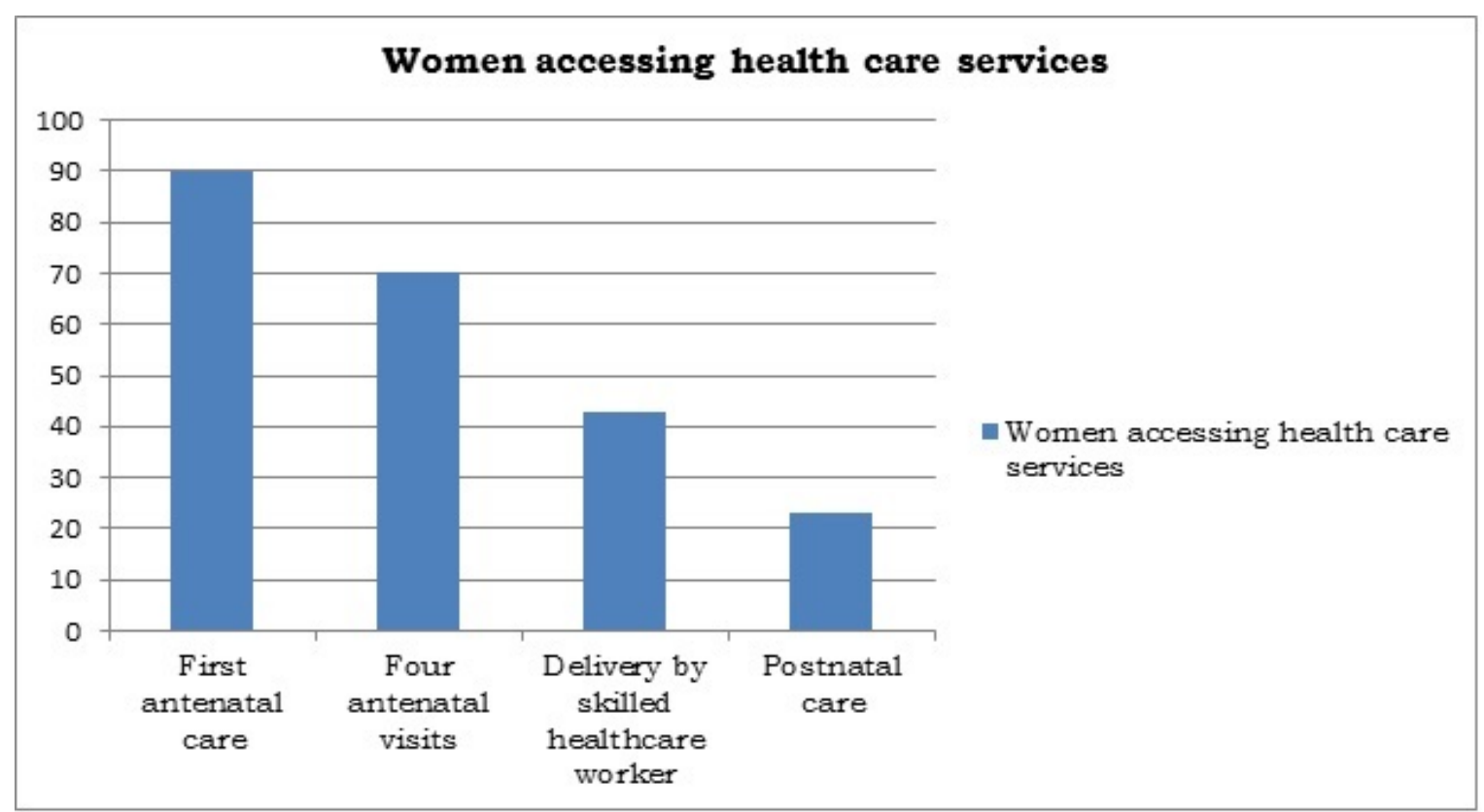

Figure 1. Women accessing health care services: current Zambian situation

As high as $90 \%$ pregnant women access $1{ }^{\text {st }}$ Antenatal attendance in company with their spouses. The number gradually drop to below $70 \%$ of those accessioning four visits and only about half end up delivering at the facility.

\section{Conclusion}

Analysis of study evidences has shown significant association between male partner involvement in birth preparedness, skilled antenatal attendances, and women's decision-making on location of birth, assistance by SBAs and outcomes of pregnancy. Evidences showed that women who take individual decision on location of birth are more likely not to deliver under the assistance of SBAs (Skilled Birth Attendants). Male involvement in decision-making increases the likelihood of women having attendance by SBAs at birth. It was established that women who received education with their husbands were more likely to make adequate childbirth preparations than those who received the education alone. The study further established that educating women together with their partners resulted into likelihood of women receiving not only skilled delivery services but also postpartum care and increase involvement of male partners in ANC services. Based on these evidences presented, this study recommends that the maternal health care programmes, therefore, need to shift from the current policy of only targeting women to promoting increased male partner involvement in safe motherhood programmes.

\section{References}

[1]. Advanced health Management Programme (2012) Leadership .Pretoria. Foundation for professional Development

[2]. Bruijn, B (2004). Perspectives on men: the nexus of policy, theory and research. Amsterdam. Netherlands Interdisciplinary Demographic Institute.

[3]. Central Statistical Office (2007) Zambia Demographic and health Survey. Lusaka. Government Printers [4]. Centre for Reviews and Dissemination (2008) Systematic reviews: CRD's Guidance for undertaking reviews in health care, January, University of York.

[5]. Duda, MB (2002) Capacity building in reproductive health programmes focussing on male involvement: a south to south framework. Washington DC. World Health Organisation.

[6]. Groenewold, G. Horstman, R and Bruijn, B (2004). Gender and the roles of men in reproductive health: Application in studies on HIV sexual risk behaviour in Zambia, Safe motherhood in Nepal. Amsterdam. Netherlands Interdisciplinary Demographic Institute. 
Texila International Journal of Public Health

Volume 5, Issue 2, Jun 2017

[7]. Horstman, R (2004). Role of husbands in Maternal Health in Morang District, Nepal. Amsterdam. Netherlands Interdisciplinary Demographic Institute.

[8]. Iliyasu et al (2010) Birth Preparedness, Complication Readiness and Fathers' Participation in Maternity Care in a Northern Nigerian Community. African Journal of Reproductive Health, 14 (1): 21-32

[9]. JHPIEGO (2004). Monitoring birth preparedness and complication readiness tools and indicators for maternal and new born health. Maryland, JHPIEGO.

[10]. Kabakyenga et al (2012) Influence of Birth Preparedness, Decision-Making on Location of Birth and Assistance by Skilled Birth Attendants among Women in South-Western Uganda. PLoS ONE, 7 (4), 1 - 8.

[11]. Kakaire et al (2011) Male involvement in birth preparedness and complication readiness for emergency obstetric referrals in rural Uganda. Reproductive Health, 8 (12), 2-7

[12]. Kinanee, J, B and Ezekiel-Hart, J (2009) Men as partners in maternal health: Implications for reproductive health counselling in Rivers State, Nigeria. Journal of Psychology and Counseling, 1 (3), 039044

[13]. Kululanga et al (2011) Striving to promote male involvement in maternal health care in rural and urban settings in Malawi - a qualitative study. Reproductive Health, 8 (36), 2-12

[14]. Lundazi District Management Team (2010) Health information management system. Lundazi District Health Office.

[15]. Mehta, M (2001). Communicating with men to promote family planning: Lessons learnt and suggestions for programming. Programme for male involvement in reproductive health. Washington DC, World Health Organisation

[16]. Ministry of Health (2010). Campaign for Accelerated Reduction of Maternal Mortality in Africa. Lusaka. Ministry of Health

[17]. Mullany et al (2007). The impact of including husbands in antenatal health education services on maternal health practices in urban Nepal: results from a randomized controlled trial. Health Education Research, 22 (2) 166-176.

[18]. Mullany et al (2009) Impact of Husbands' Participation in Antenatal Health Education Services on Maternal Health Knowledge. Journal of Nepal Medical Association, 48 (173), 28-34.

[19]. Nanjala, M and Wamalwa, D. (2012) Determinants of Male Partner Involvement in Promoting Deliveries by Skilled Attendants in Busia, Kenya. Global Journal of Health Science, 4 (2), 60 - 67.

[20]. NHS Leadership Academy (2011) Leadership Framework. London: NHS Institute for Innovation \& Improvement

[21]. Ntabona, A B (2001). Involving men in safe motherhood: the issues. Programme for male involvement in reproductive health. September, pp.54- 57.

[22]. Nzioka, C (2001). Research on men and its implications on policy and programme development in reproductive health. Washington DC, World Health Organisation.

[23]. Pandelides, E A (2001) Male involvement in prevention of pregnancy and HIV infection: results for research in four Latin American cities. Programme for male involvement in reproductive health. September, pp.15-25.

[24]. Shija et al (2011). Maternal health in fifty years of Tanzania independence: challenges and opportunities of reducing maternal mortality. Tanzania journal of health research, 13 (1), 1-14

[25]. Sternberg, P and Hubley, J (2004) Evaluating men's involvement as a strategy in sexual and reproductive health promotion. Health promotion international, 19 (3), 389-396

[26]. Story et al (2012) Husbands' involvement in delivery care utilization in rural Bangladesh: A qualitative study. BMC Pregnancy and Childbirth, 12 (28), $2-12$.

[27]. Tweheyo et al (2010) Male partner attendance of skilled antenatal care in peri-urban Gulu district, Northern Uganda .BMC Pregnancy and Childbirth, 10(53), 2-9.

[28]. World Health Organisation (2004). Beyond the numbers: Reviewing Maternal Deaths and Complications to make pregnancy safer. Geneva. World Health Organisation.

[29]. World health Organisation (2007) Fatherhood and health outcomes in Europe. A review of fatherhood and reproductive health. pp, 1-6.

[30]. World Health Organisation (2010). Working with individuals, families and communities to improve maternal and new born health. Geneva. World Health Organisation 
Texila International Journal of Public Health Volume 5, Issue 2, Jun 2017

[31]. Wynter, H. H and Hamilton, P.I (2001). Male participation in reproductive health - a Caribbean imperative. September, pp. 103114. 